\title{
A caution regarding use of the hint procedure to determine whether partial stimulus information activates responses
}

\author{
ROBERT W. PROCTOR and T. GILMOUR REEVE \\ Auburn University, Auburn, Alabama
}

\begin{abstract}
Two experiments examined the hint procedure that Miller (1983) developed to determine whether response preparation begins before stimulus recognition finishes. Miller obtained an underadditive hint $\times$ discriminability interaction for reaction times to two-dimensional, symbolic stimuli assigned to the index and middle fingers of each hand. He interpreted this interaction as indicating that response preparation begins when the first stimulus dimension is identified. The present experiments failed to replicate the underadditive interaction for reaction times in three situations that met the logical requirements for producing the interaction. In Experiment 2, error rates did show an underadditive hint $\times$ discriminability interaction, but detailed analyses of the specific incorrect responses implicated stimulus-response translation processes rather than responsepreparation processes. Because the results obtained with the hint procedure arise from nonmotoric processes, we recommend caution regarding use of the procedure to determine whether partial stimulus information activates responses.
\end{abstract}

Continuous models of human information processing indicate that partial stimulus information should activate responses (e.g., Eriksen \& Schultz, 1979), whereas discrete-stage models indicate that it should not (e.g., Sternberg, 1969). Intermediate models allow preliminary activation of responses only if the partial stimulus information is a discrete code (e.g., Miller, 1982). Therefore, the alternative models differ in whether response preparation on the basis of partial stimulus information is possible, and if so, in whether discrete stimulus codes are necessary for such preparation to occur.

To determine whether responses are activated by partial stimulus information requires (1) a phenomenon that is attributable to response preparation and (2) use of the phenomenon as a criterion response-preparation effect for situations in which partial information could be available. Miller (1982) obtained a phenomenon that appeared to satisfy the first requirement: when pairs of responses from the index and middle fingers of each hand were precued, a precuing benefit occurred only for responses on the same hand, thus producing a same-hand advantage. This advantage was then used as the criterion responsepreparation effect for a partial-information procedure that evaluated the nature of processing for a variety of stimulus sets. The same-hand advantage was found for stimuli that were composed of two distinct dimensions (e.g., letter identity and size), but only when the more salient dimension distinguished between hands. Miller interpreted these and other results as supporting an intermediate

We would like to thank James McAlarney III and Mei-Shio Jang for programming Experiments 1 and 2, respectively. We would also like to express our gratitude to Tom Childers for assistance in conducting the study. Requests for reprints should be sent to Robert W. Proctor, Department of Psychology, Auburn University, Auburn, AL 36849. model in which response preparation begins after recognition processes have activated a component code of the stimulus (the asynchronous discrete coding model).

Miller (1983) modified the partial-information procedure to provide an additional test of his asynchronous discrete coding model. This new procedure, which we shall refer to as the hint procedure, was intended to allow different amounts of time for same-hand responses to be prepared. The opportunity for differential preparation was created through the use of stimuli that varied in both letter identity and size. There were four letter identities, each of which was presented in two sizes. Two of the letter identities were assigned to each hand, with the larger size of each letter assigned to the rightmost finger of the appropriate hand (the left-hand index finger or the right-hand middle finger) and the smaller size assigned to the leftmost finger (the left-hand middle finger or the right-hand index finger; see Figure 1 for an example using the stimuli from the present Experiment 1).

The amount of time for preparation of same-hand responses was varied by making the size discrimination difficult for one of the two letters assigned to a hand (the low-discriminability condition) and easy for the other letter assigned to that hand (the high-discriminability condition). The basic idea behind the discriminability manipulation was that advance preparation of responses on the basis of letter identity could occur more efficiently when the size discrimination was difficult than when it was easy. This idea was based on the assumption of Miller's (1983) asynchronous discrete coding model that the additional time required for the size discrimination provides a greater opportunity for identification and use of the letter code.

The hint procedure employed a precue (hint) condition to show the response preparation based on partial stimu- 


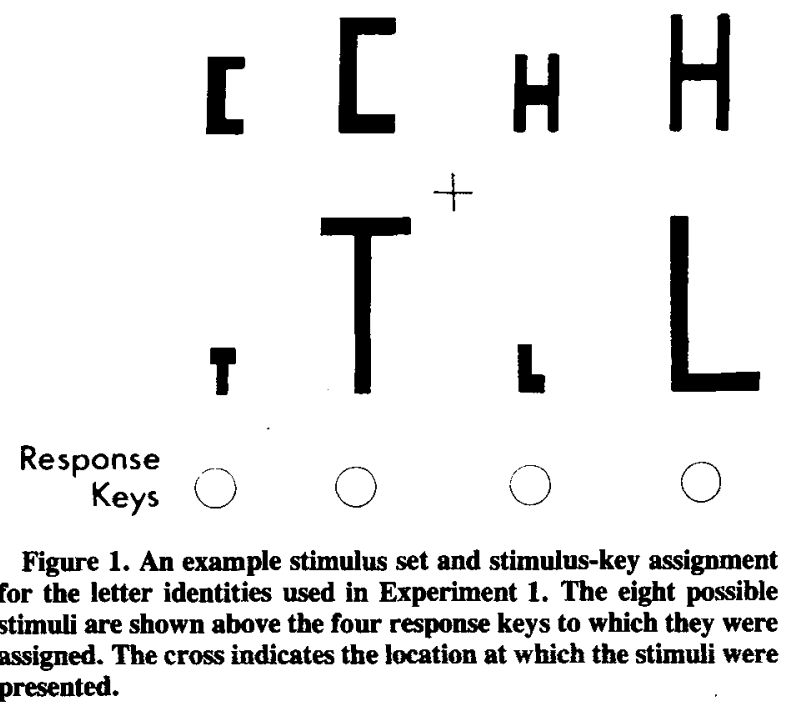

lus information. According to the asynchronous discrete coding model, the difficult size discrimination should benefit less from the hint than should the easy size discrimination (i.e., there should be an underadditive hint $x$ discriminability interaction). This underadditive interaction should occur because the difficult size discrimination allows more time for response preparation from the letter-identity information than does the easy size discrimination. That is, without an overt hint, considerable advance response preparation based on letter identity should occur for the difficult size discrimination because of the additional time required to make this discrimination. Since more advance response preparation occurs for the difficult size discrimination than for the easy size discrimination when there is no hint, the additional response preparation due to the hint should be less for the former discrimination than for the latter.

The test of the asynchronous discrete coding model using the hint procedure obtained the predicted underadditive hint $X$ discriminability interaction for reaction times (Miller, 1983, Experiment 1). Further experiments also produced results generally consistent with the asynchronous discrete coding model; that is, the underadditive hint $x$ discriminability interaction was found only for stimulus sets composed from two distinct dimensions.

The logic of both the partial-information procedure (Miller, 1982) and the hint procedure (Miller, 1983) requires that the criterion measure used to evaluate the role of partial stimulus information in response preparation be a response-preparation effect. Recently, we obtained evidence that the criterion measure for the partial-information procedure, the same-hand advantage, is a function of nonmotoric processes, rather than of motoric responsepreparation processes (Proctor \& Reeve, 1984, 1985; Reeve \& Proctor, 1984). In our experiments, a precuing advantage was obtained when the advance information specified the two leftmost or the two rightmost response locations. When the hands were placed in a normal, ad- jacent manner, as in Miller's (1982) study, the advantage corresponded to the hand distinction. However, when the hands were overlapped, so that the fingers from the two hands were alternated, the advantage occurred for fingers on different hands. Thus, the "same-hand" advantage was obtained for the fingers assigned to the two left and two right locations, regardless of whether the fingers were from the same hand.

The importance of the spatial relationships in determining the precuing advantage suggests that the advantage has its basis in stimulus-response translation processes (Nicoletti, Anzola, Luppino, Rizzolatti, \& Umiltà, 1982; Reeve \& Proctor, 1985; see Miller, 1985, for an opposing view). These translation processes operate according to a salient-features coding principle (Proctor \& Reeve, $1985,1986)$. For horizontal response locations, the response set is coded in terms of whether the locations are to the left or right of center. This spatial coding of the response set occurs with both spatial-location stimuli (Reeve \& Proctor, 1984) and two-dimensional symbolic stimuli (Proctor \& Reeve, 1985). The precuing advantage for the two left and two right response locations is obtained when a salient feature of the stimulus set corresponds to the salient left-right feature of the response set, thus allowing the translation processes to operate most rapidly for those assignments.

Because the criterion measure for Miller's (1982) partial-information procedure (i.e., the same-hand advantage) is attributable to stimulus-response translation processes, rather than to response-preparation processes, the measure does not provide evidence regarding the nature of response activation. If the criterion measure for Miller's (1983) hint procedure (i.e., the underadditive hint $x$ discriminability interaction) also were a function of translation processes, then the results obtained with this measure again would not provide evidence regarding discrete versus continuous response activation. The present experiments were intended to determine whether the underadditive interaction for the hint procedure is due to translation processes or to response-preparation processes. The experiments did not replicate the underadditive interaction for reaction times that Miller (1983) obtained, even though the situations met the necessary requirements to do so (see Miller, 1983, p. 164). However, Experiment 2 did show an underadditive hint $x$ discriminability interaction for error rates. Detailed analyses of the error patterns indicated that stimulus-response translation processes were the source of the interaction. Implications of the experimental results for the use of the hint procedure are discussed after presentation of the experiments.

\section{EXPERIMENT 1}

\section{Method}

Apparatus and Stimuli. Stimuli were presented on the display screen of a Radio Shack TRS-80 Model 4 microcomputer. Stimulus displays were software-generated block-letter versions of the letters $C, H, L$, and $T$ in four different sizes. The stimuli were white 
against a dark background, with the stroke width of the component lines being $.30^{\circ}$ when viewed by the subjects from approximately $50 \mathrm{~cm}$.

Responses were made with the middle and index fingers of each hand, which were placed on the two leftmost and two rightmost keys on the bottom row of the keyboard (the Z, X, ., and / keys). Stimulus durations and intervals were timed by the computer, and response locations and reaction times were recorded by it. The four stimulus sizes subtended visual angles of approximately $.69^{\circ} \times$ $.92^{\circ}, 1.03^{\circ} \times 1.37^{\circ}, 1.37^{\circ} \times 2.06^{\circ}$, and $1.95^{\circ} \times 3.09^{\circ}$ in width and height, respectively. The stimuli spanned a larger range of sizes than that used by Miller; this difference was necessary because our computer was not equipped with a special graphics package. The assignment of stimuli to responses was similar to that of Miller (1983; see Figure 1 of the present article). Letter identity specified hand, with two letters assigned to each hand, and stimulus size specified the appropriate finger on the hand. For any letter, the smaller size was assigned to the leftmost response on the relevant hand (i.e., the left middle finger or the right index finger) and the larger size was assigned to the rightmost response (i.e., the left index finger or the right middle finger). For one letter on each hand, the two sizes were of high discriminability $\left(.92^{\circ}\right.$ and $\left.3.09^{\circ}\right)$, whereas for the other letter the sizes were of low discriminability $\left(1.37^{\circ}\right.$ and $2.06^{\circ}$ ). The specific assignments of letters to hands and to discriminability conditions were counterbalanced across subjects.

A warning stimulus occurred prior to, and in the same location as, the target stimulus. In the no-hint condition, the warning stimulus was a diamond-shaped figure that was uninformative about the target stimuli that were possible for that trial. That is, with this neutral warning stimulus, any of the eight target stimuli could occur. In the hint condition, the warning stimulus was an arrow that pointed either to the left side or to the right side of the display. The arrow indicated that the target stimulus would be one of the four stimuli assigned to the side of the keyboard to which the arrow pointed.

Subjects and Procedure. The subjects were 32 students enrolled in undergraduate psychology courses at Auburn University who participated for extra credit. Each subject was tested in a single experimental session, during which he or she received four blocks of 80 trials (the first block was practice). Within each block, each target stimulus was presented five times in the hint condition and five times in the no-hint condition. A trial began with a 750-msec presentation of either the informative hint or the neutral warning signal, followed by a 750 -msec blank period. At the end of this period a single target stimulus was displayed until the subject responded. If the response was correct, the next trial began after a 1-sec delay. If the response was incorrect, the entire set of stimulus letters was displayed in a matrix on the screen, with the corresponding responses indicated (the display was similar to Figure 1). This display remained in view until the subject pressed the space bar, at which time the sequence for the next trial began.

Subjects were shown all eight stimulus forms, along with the particular stimulus-response assignments, before the start of the experiment. The instructions emphasized that the smaller size for each letter was assigned to the leftmost finger on the appropriate hand. Subjects were told that the hint indicated the correct hand for the response and that the hint would never be misleading. The instructions stressed responding as quickly as possible without making too many errors.

\section{Results and Discussion}

Mean correct reaction times and proportions of errors were computed for each subject as a function of size discriminability (high, low) and hint condition (hint, no hint). Responses faster than $200 \mathrm{msec}$ or slower than $2,000 \mathrm{msec}$ (less than $1 \%$ of the responses) were excluded
Table 1

Mean Reaction Time (in Milliseconds) and Proportion of Errors as a Function of Discriminability and Hint Condition in Experiment 1

\begin{tabular}{lcccccc}
\hline & \multicolumn{2}{c}{ Reaction Time } & & \multicolumn{2}{c}{ Proportion of Errors } \\
\cline { 2 - 3 } \cline { 6 - 7 } & High & Low & & High & Low \\
\hline No-Hint Condition & 744 & 860 & & .083 & .124 \\
Hint Condition & 587 & 698 & & .022 & .060 \\
Hint Effect & 157 & 162 & & .061 & .064 \\
Interaction & \multicolumn{2}{c}{+5} & & \multicolumn{2}{c}{+.003} \\
\hline
\end{tabular}

from the computation of the mean reaction times. The data were averaged across subjects, and the results are shown in Table 1.

Responses were faster and more accurate to the targets from the high-discriminability pairs (means $=666 \mathrm{msec}$ and .053) than to the targets from the low-discriminability pairs (means $=779 \mathrm{msec}$ and .092$)\left[F_{\mathrm{s}}(1,31) \geq 53.5\right.$, $p s<.001]$. Also, responses were faster and more accurate for the hint condition (means $=643 \mathrm{msec}$ and .041 ) than for the no-hint condition (means $=802 \mathrm{msec}$ and .104) $[F \mathrm{~s}(1,31) \geq 16.1, p \mathrm{~s}<.001]$. Therefore, both a discriminability effect and a hint effect were obtained. Most importantly, the reaction times, as well as the errors, did not show a hint $\times$ discriminability interaction $(F \mathrm{~s}<1)$, indicating that the benefit of the hint was equivalent for the high- and low-discriminability conditions.

Therefore, Experiment 1 failed to replicate the underadditive hint $\times$ discriminability interaction for reaction times obtained by Miller (1983). This failure to replicate the interaction occurred despite the fact that we followed Miller's method closely. The primary deviation from his method was only that our stimuli varied over a larger range of sizes than did his. However, the range of sizes should not be crucial, because, according to the logic of the hint procedure, "the amount of response preparation using preliminary information should be positively related to the difficulty of the secondary discrimination" (Miller, 1983 , p. 164). In the present experiment, a substantial discriminability effect was obtained for the secondary size dimension when there was no hint (responses were $116 \mathrm{msec}$ faster to targets from high-discriminability pairs). Because this size discriminability effect was greater than the 62-msec effect shown by Miller, there should have been at least as much opportunity in the present experiment for responses to be activated on the basis of letter identity when discriminability was low.

\section{EXPERIMENT 2}

There are two possible reasons for the failure to replicate the underadditive hint $\times$ discriminability interaction for reaction times in Experiment 1. First, the difference between the range of stimulus sizes used in our experiment and the range used in Miller's (1983) study may be crucial, even though according to the logic of the hint procedure it should not be. Second, the underadditive interaction for reaction times may not be a robust finding. 
That is, the interaction may not be obtained reliably even when the range of stimulus sizes more closely approximates that used by Miller.

Experiment 2 was designed to distinguish between these two possibilities. One group of subjects was tested with stimuli whose four sizes approximated those of the stimuli used by Miller (1983). For a second group of subjects, the stimuli were doubled in size so that the four sizes were similar to those used in Experiment 1. Moreover, by doubling the stimulus sizes, the ratios of the four stimulus sizes were the same for both stimulus sets.

\section{Method \\ Apparatus and Stimuli. Stimuli were presented on the display screen of a Radio Shack TRS-80 Model III microcomputer, for which the display screen and keyboard are similar to those of the Model 4 used in Experiment 1. The Model III microcomputer was used because it was equipped with a graphics package that permit- ted a wider range of stimulus sizes than was used in Experiment 1. The stroke width of the stimuli used in this experiment was approx- imately $.12^{\circ}$ of visual angle, which is thin relative to the stroke width of $.30^{\circ}$ used in Experiment 1.}

Again, the stimuli were letters of different sizes. However, an expanded pool of letter identities and two sets of sizes were used. The pool of letter identities included C, E, F, H, I, K, L, N, T, $U, X$, and $Z$. The two sets of sizes differed only in that the heights and widths of one set (the double-size set) were twice those of the other set (the standard-size set). The visual angles subtended by the four sizes in the standard-size set were approximately $.36^{\circ} \times$ $.64^{\circ}, .55^{\circ} \times .91^{\circ}, .73^{\circ} \times 1.27^{\circ}$, and $.91^{\circ} \times 1.81^{\circ}$ in width and height, respectively. The heights of these letters corresponded closely to the heights of $.65^{\circ}, .95^{\circ}, 1.24^{\circ}$, and $1.67^{\circ}$ that were used by Miller (1983; widths were not reported for his experiment). The visual angles for the corresponding sizes in the double-size set were approximately $.72^{\circ} \times 1.28^{\circ}, 1.10^{\circ} \times 1.82^{\circ}, 1.46^{\circ} \times 2.54^{\circ}$, and $1.82^{\circ} \times 3.62^{\circ}$. The double-size set were similar in size to the stimuli used in Experiment 1, with the exception of the difference in stroke width noted above. The informative and neutral warning stimuli were similar to those used in Experiment 1, and the response locations were the same as those used previously.

Each subject received only four letter identities, two assigned to the left hand and two to the right hand. The letters were presented in either the standard size or the double size, with each subject receiving only one set size. The same procedure for assigning stimuli to responses was used for both the standard-size and double-size sets, and this procedure was the same as that used in Experiment 1; that is, one of the two letters assigned to a hand was presented in only the two extreme sizes for the appropriate set and the other letter was presented in only the two intermediate sizes. For each letter, the smaller of the two sizes was assigned to the leftmost response on the appropriate hand and the larger was assigned to the rightmost response. The specific letters, and the assignments of these letters to the left or right hand and to the high- or lowdiscriminability sizes, were determined randomly for each subject. The only constraints on the randomizations were that across subjects (1) all letters were used equally often, (2) a specific set of letters assigned to hand and discriminability conditions for a subject who received the standard-size set was also used for a subject who received the double-size set, and (3) no two subjects within a set size received the exact same letters and assignments.

Subjects and Procedure. The subjects were 64 students from the same subject pool as was used in Experiment 1. None of the subjects had participated in that experiment. Half of the subjects were assigned randomly to the standard-size stimulus set and half to the double-size set. In all other respects, the procedure was the same as that used in Experiment 1.

\section{Results and Discussion}

Reaction times and proportions of errors. Mean correct reaction times and proportions of errors were computed for each subject in the same manner as in Experiment 1 . The data were averaged across subjects, and the summary means are shown in Table 2 as a function of discriminability (high, low), hint condition (hint, no hint), and set (standard size, double size).

Reaction times and accuracies did not differ reliably between the standard-size set and the double-size set $(F \mathrm{~s} \leq 1)$. Responses were faster and more accurate to stimuli from the high-discriminability pairs (means = $803 \mathrm{msec}$ and .046) than to stimuli from the lowdiscriminability pairs (means $=931 \mathrm{msec}$ and .061 ) $[F \mathrm{~s}(1,62) \geq 14.8, p \mathrm{~s}<.001]$. A significant set $\times$ discriminability interaction was present for both the reactiontime and error data $\left[F_{\mathrm{s}}(1,62) \geq 5.14, p \mathrm{~s}<.05\right]$, indicating that the differences between the low- and highdiscriminability pairs were less for the standard-size set (differences $=106 \mathrm{msec}$ and .007 ) than for the doublesize set (differences $=150 \mathrm{msec}$ and .023 ).

Responses were faster and more accurate for the hint condition (means $=741 \cdot \mathrm{msec}$ and .030 ) than for the nohint condition (means $=993 \mathrm{msec}$ and .077$)[F \mathrm{~s}(1,62)$ $\geq 83.8, p \mathrm{~s}<.001]$. For the reaction-time data, no other terms were significant. Of most importance, the reaction times showed neither a hint $\times$ discriminability interaction nor a three-way interaction of these variables with set $\left(F_{\mathrm{s}}<1\right)$. In other words, the high- and lowdiscriminability pairs showed approximately equivalent benefits of the hint on reaction times for the standard-size and double-size stimulus sets. Therefore, the underadditive interaction for reaction times reported by Miller (1983) failed to occur not only for the double-size set, which was similar to that used in our Experiment 1, but also for the standard-size set, which was similar to that used by Miller. This outcome indicates that the failure to replicate the hint $\times$ discriminability interaction for reaction times in Experiment 1 was not due to the stimulus sizes that were used.

Although an underadditive hint $\times$ discriminability interaction was not obtained for the reaction-time data, such

Table 2

Mean Reaction Time (in Milliseconds) and Proportion of Errors as a Function of Discriminability, Hint Condition, and Set in Experiment 2

\begin{tabular}{|c|c|c|c|c|}
\hline & \multicolumn{2}{|c|}{ Reaction Time } & \multicolumn{2}{|c|}{ Proportion of Errors } \\
\hline & High & Low & High & Low \\
\hline \multicolumn{5}{|c|}{ Standard-size Set } \\
\hline No-Hint Condition & 935 & 1,039 & .075 & .071 \\
\hline Hint Condition & 686 & 794 & .016 & .035 \\
\hline Hint Effect & 249 & 245 & .059 & .036 \\
\hline Interaction & \multicolumn{2}{|c|}{-4} & \multicolumn{2}{|c|}{-.023} \\
\hline \multicolumn{5}{|c|}{ Double-size Set } \\
\hline No-Hint Condition & 923 & 1,075 & .074 & .086 \\
\hline Hint Condition & 668 & 815 & .017 & .051 \\
\hline Hint Effect & 255 & 260 & .057 & .035 \\
\hline Interaction & \multicolumn{2}{|c|}{+5} & \multicolumn{2}{|c|}{-.022} \\
\hline
\end{tabular}


an interaction was evident for the error-rate data $[F(1,62)=7.97, p<.01]$. Unlike in Experiment 1, the high-discriminability pairs showed a greater benefit of the hint than did the low-discriminability pairs (see Table 2). As indicated by a nonsignificant hint $\times$ discriminability $\times$ set interaction $(F<1)$, this pattern held for both the standard-size and double-size stimulus sets. One interpretation of the hint $\times$ discriminability interaction is that the error pattern reflects the same basic interaction as that obtained by Miller (1983) for reaction times, with the interaction reflecting preparation of responses based on partial stimulus information. A detailed analysis of the incorrect responses was performed to distinguish between this response-preparation interpretation and alternative accounts.

Incorrect responses. When stimuli are two-dimensional symbolic forms, as in the present experiment, the manner in which the stimulus dimensions are assigned to the response locations is important (Proctor \& Reeve, 1985). In the present experiment, letter identity distinguished between hand locations, and size distinguished between finger locations within a hand. Therefore, incorrect responses can be categorized according to the relationship of the incorrect-response location to the correct hand and finger locations. The three categories of relationships involve responses with (1) the correct hand location, but the incorrect finger location (a finger-location error; e.g., responding with the right index finger when the correct response would be with the right middle finger), (2) the incorrect hand location, but the correct finger location (a hand-location error; e.g., responding with the left index finger when the correct response would be with the right middle finger), and (3) the incorrect hand location and the incorrect finger location (a hand-and-finger location error; e.g., responding with the left middle finger when the correct response would be with the right middle finger).

For each correct-response location, the incorrect responses were classified according to the three error categories (error types). An analysis of variance showed a significant main effect for error type $[F(2,124)=58.7$, $p<.001]$. This effect was due to the fact that the number of hand-and-finger location errors $(T=16)$ was negligible relative to the number of hand-location errors $(T=398)$ and the number of finger-location errors $(T=$ 404). Incorrect responses were divided approximately equally between responses with the correct hand (but wrong finger; the finger-location errors) and responses with the incorrect hand (the hand-location errors plus the hand-and-finger location errors). The much greater number of hand-location errors than hand-and-finger location errors indicates that when subjects responded with a finger on the wrong hand, the finger almost always corresponded to the relative left-right spatial location of the appropriate finger on the correct hand. Rarely was the incorrect response made with the anatomically equivalent finger on the wrong hand.

The finding that hand-location errors, rather than handand-finger location errors, predominated when subjects responded with the wrong hand is important because it distinguishes between the most common models of response-preparation and stimulus-response translation processes. Generally, models of response preparation propose that preparation processes involve the specification of anatomical features, such as hand (left, right) and finger (index, middle) (Miller, 1982; Rosenbaum, 1983; Rosenbaum \& Kornblum, 1982; but see Miller, 1985). Because hand-and-finger-location errors involve misspecification of only one anatomical feature (hand), whereas hand-location errors involve misspecification of two anatomical features (hand and finger), errors of the former type should outnumber those of the latter type if the errors are due to response-preparation processes. In contrast, because most models of stimulus-response translation propose that response locations are coded spatially (Nicoletti et al., 1982; Proctor \& Reeve, 1985, 1986), hand-location errors should outnumber hand-and-finger location errors if the errors are due to translation processes. The fact that hand-location errors predominate, therefore, strongly suggests that the errors arise from spatially coded stimulus-response translation processes, rather than from anatomically coded response-preparation processes.

Because hand-and-finger location errors were rare, the remaining analyses were performed only on the handlocation and finger-location errors. The numbers of errors for each of these two categories are shown in Table 3 as a function of discriminability, hint condition, and correct response. Although no overall difference existed between the number of hand-location errors and the number of finger-location errors $(F<1)$, error type interacted significantly with hint condition $[F(1,62)=36.2$, $p<.001]$ and with discriminability $[F(1,62)=73.0$, $p<.001]$. The error type $\times$ hint condition interaction reflects the fact that more hand-location errors than fingerlocation errors ( 355 vs. 223 ) were made when there was no hint, but fewer hand-location errors than fingerlocation errors ( 43 vs. 181) were made when there was a hint. The relative lack of hand-location errors in the hint condition is expected because the hint indicated the correct hand in advance.

The error type $x$ discriminability interaction is due to the fact that hand-location errors outnumbered finger-

Table 3

Total Number of Hand-Location and Finger-Location Errors in Experiment 2 as a Function of Discriminability, Hint Condition, and Correct Response

\begin{tabular}{|c|c|c|c|c|c|c|c|c|}
\hline \multirow[b]{3}{*}{ Error Type } & \multicolumn{8}{|c|}{ Discriminability } \\
\hline & \multicolumn{4}{|c|}{ High } & \multicolumn{4}{|c|}{ Low } \\
\hline & $\mathbf{L M}$ & $\mathbf{L I}$ & $\mathbf{R I}$ & $\mathbf{R M}$ & LM & LI & $\mathbf{R I}$ & $\mathbf{R M}$ \\
\hline \multicolumn{9}{|c|}{ No Hint } \\
\hline Hand & 41 & 71 & 87 & 19 & 26 & 39 & 52 & 20 \\
\hline Finger & 14 & 13 & 10 & 27 & 46 & 31 & 24 & 58 \\
\hline \multicolumn{9}{|c|}{ Hint } \\
\hline Hand & 7 & 6 & 13 & 2 & 3 & 4 & 5 & 3 \\
\hline Finger & 6 & 3 & 5 & 20 & 26 & 40 & 34 & 47 \\
\hline
\end{tabular}

Note-LM = left middle finger, $\mathrm{LI}=$ left index finger, $\mathrm{RI}=$ right index finger, and $\mathbf{R M}=$ right middle finger. 
location errors for the high-discriminability pairs ( $246 \mathrm{vs.}$ 98 ), but not for the low-discriminability pairs (152 vs. 306). This pattern was apparent for both the standard-size set and the double-size set, although the magnitude was slightly greater for the former set [error type $\times$ discriminability $\times$ set, $F(1,62)=4.01, p<.05]$. That relatively more finger-location errors were made for the lowdiscriminability pairs is in accord with the fact that the size discrimination determined whether the response should be the left or right finger location on the hand. That is, the more difficult size discrimination for the lowthan for the high-discriminability pairs caused greater confusability about finger location.

The effects of discriminability and hint condition on the type of errors are consistent with Miller's (1982) asynchronous discrete coding model. That is, the model predicts relatively fewer finger-location errors for the high-discriminability pairs than for the lowdiscriminability pairs and relatively fewer hand-location errors in the hint condition than in the no-hint condition. However, these interaction effects are not strong tests of the model, because the effects cannot be attributed clearly to response-preparation processes. More likely, since the primary influence of the discriminability and hint variables should be on stimulus identification and stimulusresponse translation processes, these nonmotoric processes are the bases for the effects.

The relative absence of hand-and-finger location errors, as noted previously, provides evidence that translation processes are involved in the selection of incorrect responses. Additional evidence for translation processes is apparent when the patterns of hand-location and fingerlocation errors are examined for each correct-response position. Error type interacted with correct response $[F(3,186)=17.5, p<.001]$, as well as with both correct response and hint $[F(3,186)=9.67, p<.001]$ (see Figure 2). These interactions indicate that hand-location errors predominated for the two inner correct-response positions but not for the two outer correct-response positions. However, this pattern was evident only for the nohint condition.

As stated previously, hand-location errors should be a factor only in the no-hint condition, as was found, because the hint specified the correct hand. That handlocation errors predominated in the no-hint condition only for the two inner correct-response positions can be explained as follows. For the inner positions, the left-right specification of hand (indicated by letter identity) was inconsistent with the left-right specification of finger (indicated by size). For example, when the right index finger was the correct response, letter identity specified the right hand and size specified the left finger on that hand. Subjects most often responded incorrectly by using the left finger on the left hand (i.e., the left middle finger), which indicates that they selected a hand location that was consistent with the correct finger location.

For the two outer correct-response positions, the leftright specification of hand was consistent with the left-

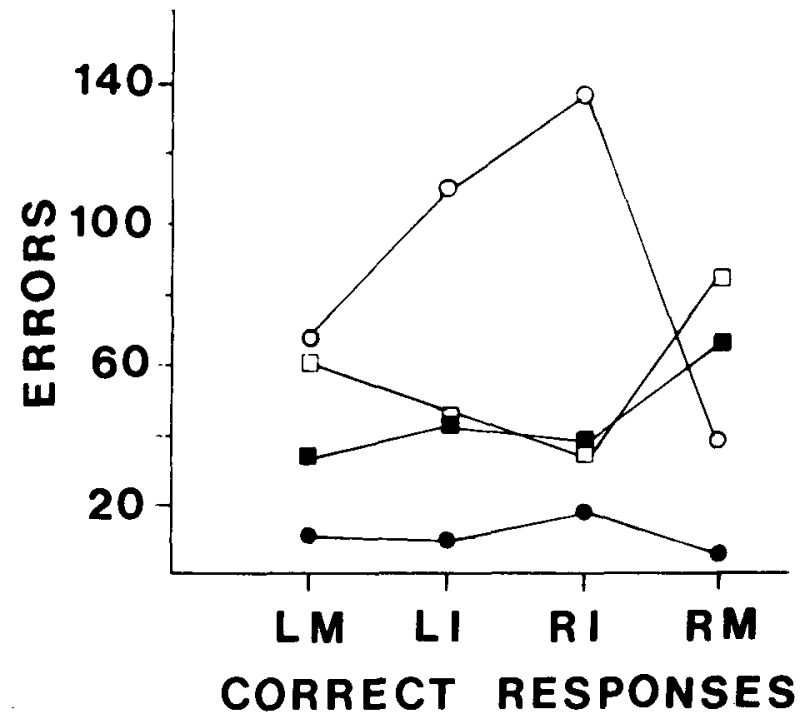

Figure 2. The total number of hand-location and finger-location errors, as a function of hint condition and correct response. $L M$ = left middle finger; $L I=$ left index finger; $R I=$ right index finger; RM = right middle finger. Circles indicate hand-location errors and squares indicate finger-location errors; unfilled symbols represent the no-hint condition and filled symbols represent the hint condition.

right specification of finger. For example, when the right middle finger was the correct response, letter identity specified the right hand and size specified the right finger. Because the correct hand was consistent with the correct finger location, the response was not in error if subjects selected a hand location that corresponded to the finger location.

The pattern of incorrect responses, therefore, suggests that subjects often responded incorrectly by selecting a hand location on the basis of the finger location. This selection process resulted in an error when the hand location was inconsistent with the finger location, but in a correct response when the hand and finger locations were consistent. Reliance on this finger-location information apparently occurred because the assignment of stimulus sizes to finger locations was by a systematic rule, whereas the assignment of letter identities to hand locations was not. That is, the four stimulus sizes were assigned to the finger locations so that the two smaller sizes always indicated the left finger location and the two larger sizes always indicated the right finger location. In contrast, the four letter identities were assigned arbitrarily to the left and right hand locations. Because of this arbitrary assignment, the translation processes involved in the determination of the correct hand should have been more demanding than those involved in the determination of the correct finger (e.g., Schneider \& Shiffrin, 1977). Subjects apparently responded on a significant proportion of the error trials by selecting both hand and finger locations on the basis of the easier translation between size and response location.

The preponderance of hand-location errors relative to finger-location errors when there was no hint was greater 
for the high-discriminability pairs than for the lowdiscriminability pairs. This relationship occurred because determining the correct finger location is more difficult when the sizes that must be discriminated are similar. Since it is no longer necessary to determine hand location from letter identity when a hint is provided, handlocation errors were minimal for both high- and lowdiscriminability pairs in the hint condition. Thus, relative to the no-hint condition, hand-location errors decreased more in the hint condition for the high-discriminability pairs than for the low-discriminability pairs. This relative shift in hand-location errors, which results from the translation requirements' differing in the hint and no-hint conditions, is the primary source of the underadditive hint $\times$ discriminability interaction in the error-rate data.

Although the underadditive hint $\times$ discriminability interaction was not apparent in Experiment 1, the pattern of incorrect responses in that experiment was similar to the pattern obtained in Experiment 2. That is, when there was no hint, hand-location errors outnumbered fingerlocation errors for the two inner correct-response positions, and this effect was larger for the highdiscriminability pairs than for the low-discriminability pairs. When there was a hint, few hand-location errors were made, resulting in a greater reduction in the proportion of these errors for the high-discriminability pairs. Thus, the shift in hand-location errors that produced the underadditive hint $\times$ discriminability interaction in Experiment 2 also was evident in Experiment 1. As previously noted, this shift in hand-location errors seems to have its basis in stimulus-response translation processes.

The primary difference between the patterns of incorrect responses in Experiments 1 and 2 was that the proportion of finger-location errors for the low-discriminability pairs was substantially greater in Experiment 1 . Because this difference was most pronounced for the no-hint condition, the low-discriminability pairs showed a more substantial benefit of the hint on finger-location errors than did the high-discriminability pairs. Thus, the hint's greater benefit on hand-location errors for the highdiscriminability pairs was offset in Experiment 1 by the hint's greater benefit on finger-location errors for the lowdiscriminability pairs. Therefore, whether an underadditive hint $\times$ discriminability interaction was obtained for the error-rate data was a function of whether a translation factor, which tended to produce such an interaction, was offset by a perceptual-discriminability factor that tended to counteract the underadditive interaction.

\section{Summary}

In Experiment 2, as in Experiment 1, no underadditive hint $\times$ discriminability interaction was obtained for reaction times. However, such an interaction was apparent for error rates. Detailed analyses of the incorrect responses showed that the errors were primarily a function of stimulus-response translation processes, rather than of response-preparation processes. The underadditive hint $x$ discriminability interaction that was obtained likely reflects only the different translation requirements for the hint and no-hint conditions.

\section{GENERAL DISCUSSION}

The hint procedure developed by Miller (1983) is one of several recent attempts (e.g., Coles, Gratton, Bashore, Eriksen, \& Donchin, 1985; Meyer, Yantis, Osman, \& Smith, 1985) to address the fundamental issue of whether partial stimulus information activates responses. According to the logic of the hint procedure, an underadditive hint $\times$ discriminability interaction provides evidence for response activation based on partial stimulus information. Miller obtained such an interaction for reaction times to stimuli that differed in letter identity and size. Based on this and other outcomes, he concluded that preliminary response activation occurs for stimuli composed of discrete codes.

To evaluate the relative roles of translation and responsepreparation processes, the present study reexamined the hint procedure for situations in which the stimuli differed in letter identity and size. These situations met the hint procedure's requirements for producing an underadditive hint $\times$ discriminability interaction for reaction times. Yet the interaction was not obtained in any of the situations, including one (Experiment 2, the standard-size stimulus set) for which the stimulus sizes approximated those used by Miller (1983). Experiment 2 did show an underadditive hint $x$ discriminability interaction, but for the proportions of errors rather than for reaction times. This interaction for error rates was not significant either in our Experiment 1 or in Miller's experiment. Therefore, although there is a tendency for underadditivity with the hint procedure, the interaction patterns obtained for reaction times and errors are not very stable or reliable.

The hint procedure allows the opportunity for nonmotoric processes to produce an underadditive hint $\times$ discriminability interaction. This opportunity occurs because the requirements for the hint and no-hint conditions differ both quantitatively and qualitatively. The quantitative distinction between the conditions is that the number of stimulus and response alternatives differs: there are eight stimuli and four responses when there is no hint, and four stimuli and two responses when there is a hint. Such quantitative differences in the number of stimulus-response alternatives are known to influence stimulus identification and stimulus-response translation processes (Garner, 1985; Schmidt, 1982; Teichner \& Krebs, 1974; Zelaznik, 1978).

More important is the qualitative distinction between the hint and no-hint conditions. In the no-hint condition, the subject must both identify the letter and discriminate the size to accurately determine the correct response. However, in the hint condition, only size must be discriminated, because the information provided by letter identity is redundant with the hint. That is, when there is no hint, letter identity indicates which hand is appropriate for responding, whereas size indicates the finger on the 
hand. However, when the hand is prespecified by the hint, the task becomes one of responding with the left response on the hand if the stimulus is one of the two smaller sizes and the right response if it is one of the two larger sizes, regardless of letter identity. Therefore, the stimulusresponse translation requirements differ qualitatively, as well as quantitatively, between the no-hint and hint conditions.

Detailed analyses of the incorrect responses in Experiment 2 provide evidence that translation processes are major contributors to the particular errors that are made. First, when subjects responded with the wrong hand, the response almost always involved the finger whose leftright location corresponded to that of the correct response, rather than the finger that corresponded anatomically. The use of spatial coding is a characteristic of most stimulusresponse translation models (Nicoletti et al., 1982; Proctor \& Reeve, 1985, 1986; Reeve \& Proctor, 1985), but not of most response-preparation models (Miller, 1982; Rosenbaum, 1983; Rosenbaum \& Kornblum, 1982).

Second, hand-location errors (responding with the correct finger location but on the wrong hand) outnumbered finger-location errors (responding with the incorrect finger location on the correct hand) when there was no hint, but only for the two inner response positions. The predominance of hand-location errors for the two inner positions indicates that subjects often selected the hand for responding on the basis of the finger location that was signaled by the stimulus. These hand-location errors were limited to the two inner correct-response positions because, for the two outer positions, the selection of hand on the basis of finger location resulted in a correct response. Subjects responded on some trials by determining the correct finger location and then selecting the hand according to the sarne left-right value, because the assignments of letter identities to hands were less systematic than the assignments of sizes to fingers. Such hand-location errors, by their nature, occur in the stimulus-response translation processes.

One important point regarding the translation-based hand-location errors is that they disappeared when a hint was provided. As indicated previously, the methodological reason for the decrease of hand-location errors with the hint is that discrimination between hands on the basis of letter identity is no longer required. The theoretical importance of these data is that they provide evidence for the argument made previously that the hint alters the qualitative nature of the translation processes. This evidence affirms the point that when an underadditive hint $X$ discriminability interaction is obtained for either errors or reaction times, it cannot be attributed to responsepreparation processes.

The hint procedure was designed initially to examine the processing of information between stimulusidentification processes and response-preparation processes (Miller, 1983). However, "there is typically thought to be an intervening decision process (e.g., Smith, 1968), and this process has been ignored"' (Miller, 1983, p. 181) in the interpretation of the results obtained with the procedure. The present study indicates that these decision or translation processes play a crucial role in the hint procedure and, therefore, that they should not be ignored.

Tests between continuous and discrete models of response preparation, such as the hint procedure, require the manipulation of variables whose direct influences should be on stimulus-identification and stimulus-response translation processes. These variables include stimulus discriminability, the presence or absence of a precue, and stimulus-response assignments. However, if the effects of the variables are to provide evidence regarding whether partial stimulus information activates responses, these effects must be attributed indirectly to response preparation. Because the direct influences of the variables are on nonmotoric processes, interpretations in terms of response-preparation processes must be made with caution.

\section{REFERENCES}

Coles, M. G. H., Gratton, G., Bashore, T. R., Eriksen, C. W., \& Donchin, E. (1985). A psychophysiological investigation of the continuous flow model of human information processing. Journal of Experimental Psychology: Human Perception \& Performance, 11, 529-553.

ERIKSEN, C. W., \& SChULTZ, D. W. (1979). Information processing in visual search: A continuous flow conception and experimental results. Perception \& Psychophysics, 25, 249-263.

GARNER, W. R. (1985). Contingent information processing: Contingent and precued classification. Perception \& Psychophysics, 38, 237-248.

Meyer, D. E., Yantis, S., Osman, A. M., \& SmTth, J. E. K. (1985). Temporal properties of human information processing: Tests of discrete versus continuous models. Cognitive Psychology, 17, 445-518.

MiLler, J. (1982). Discrete versus continuous stage models of human information processing: In search of partial output. Journal of $E x-$ perimental Psychology: Human Perception \& Pefformance, 8, 273-296.

MilleR, J. (1983). Can response preparation begin before stimulus recognition finishes? Journal of Experimental Psychology: Human Perception \& Performance, 9, 161-182.

MILLER, J. (1985). A hand advantage in preparation of simple keypress responses: Reply to Reeve and Proctor. Journal of Experimental Psychology: Human Perception \& Performance, 11, 221-233.

Nicoletti, R., Anzola, G. P., Luppino, G., Rizzolatti, G., \& UMILTA, C. (1982). Spatial compatibility effects on the same side of the body midline. Journal of Experimental Psychology: Human Perception \& Performance, 8, 664-673.

Proctor, R. W., \& Reeve, T. G. (1984, November). Can preparation procedures determine whether partial information activates responses? Paper presented at the 25th Annual Meeting of the Psychonomic Society, San Antonio, TX.

Proctor, R. W., \& ReEve, T. G. (1985). Compatibility effects in the assignment of symbolic stimuli to discrete finger responses. Journal of Experimental Psychology: Human Perception \& Performance, 11, 623-639.

Proctor, R. W., \& ReEve, T. G. (1986). Salient-feature coding operations in spatial precuing tasks. Journal of Experimental Psychology: Human Perception \& Performance, 12, 277-285.

Reeve, T. G., \& Proctor, R. W. (1984). On the advance preparation of discrete finger responses. Journal of Experimental Psychology: Human Perception \& Performance, 10, 541-553.

Reeve, T. G., \& Proctor, R. W. (1985). Nonmotoric translation processes in the preparation of discrete finger responses: A rebuttal 
of Miller's (1985) analysis. Journal of Experimental Psychology: Human Perception \& Performance, 11, 234-240.

RosenBaum, D. A. (1983). The movement precuing technique: Assumptions, applications, and extensions. In R. A. Magill (Ed.), Memory and control of action (pp. 231-274). Amsterdam: North-Holland.

Rosenbaum, D. A., \& Kornblum, S. (1982). A priming method for investigating the selection of motor responses. Acta Psychologica, 51, 223-243.

SCHMidT, R. A. (1982). Motor control and learning. Champaign, IL: Human Kinetics Publishers.

SCHNEIDER, W., \& SHIFFrIN, R. M. (1977). Controlled and automatic human information processing: I. Detection, search, and attention. Psychological Review, 84, 1-66.
SMITH, E. E. (1968). Choice reaction time: An analysis of the major theoretical positions. Psychological Bulletin, 69, 77-110.

STERNBERG, S. (1969). The discovery of processing stages: Extensions of Donder's method. In W. G. Koster (Ed.), Attention and performance II (pp. 276-315). Amsterdam: North-Holland.

Teichner, W. H., \& KREBS, M. J. (1974). Laws of visual choice reaction time. Psychological Review, 81, 75-98.

ZELAZNIK, H. (1976). Precuing response factors in choice reaction time: A word of caution. Journal of Motor Behavior, 10, 77-79.

(Manuscript received March 14, 1985; revision accepted for publication June 25,1986 .) 